\title{
Revisiting the Classics to Recover the Physical Sense in Electrical Noise*
}

\author{
Jose-Ignacio Izpura \\ Group of Microsystems and Electronic Materials (GMME-CEMDATIC), \\ Universidad Politécnica de Madrid (UPM), Madrid, Spain \\ E-mail: joseignacio.izpura@upm.es \\ Received March 1, 2011; revised April 17, 2011; accepted May 3, 2011
}

\begin{abstract}
This paper shows a physically cogent model for electrical noise in resistors that has been obtained from Thermodynamical reasons. This new model derived from the works of Johnson and Nyquist also agrees with the Quantum model for noisy systems handled by Callen and Welton in 1951, thus unifying these two Physical viewpoints. This new model is a Complex or 2-D noise model based on an Admittance that considers both Fluctuation and Dissipation of electrical energy to excel the Real or 1-D model in use that only considers Dissipation. By the two orthogonal currents linked with a common voltage noise by an Admittance function, the new model is shown in frequency domain. Its use in time domain allows to see the pitfall behind a paradox of Statistical Mechanics about systems considered as energy-conserving and deterministic on the microscale that are dissipative and unpredictable on the macroscale and also shows how to use properly the Fluctuation-Dissipation Theorem.
\end{abstract}

Keywords: Quantum-Compliant, Noise Model, Fluctuation-Dissipation Theorem, Cause-Effect, Action-Reaction

\section{Introduction}

Looking for the state of the art on shot noise in devices we found a publication [1] whose authors contend that electrical charge being piled-up in a pure resistance $R$ devoid of any capacitance $C$, generates shot noise. Since a pure resistance is not a noisy device accordingly to the well known work of Callen \& Welton [2] nor to the classical Fluctuation-Dissipation Theorem (FDT) derived from [2] that we find hard to apply to a pure resistance $R$, we decided to consider in some detail the academic viewpoint on the FDT given in a recent paper [3]. Here, the FDT was nicely explained by a mechanical example whose analogy with our Admittance-based model (Amodel) for electrical noise, led us to write this paper. This A-model that agreeing with [2] would be a "Quantum-compliant" model for electrical noise, excels the model in use today for resistors and has explained recently $1 / f$ excess noise in Solid-State devices [4] and flicker noise in vacuum ones [5] as simple consequences of thermal noise in these devices.

*Work supported by the Spanish CICYT under the MAT2010-18933 project, by the Comunidad Autónoma de Madrid through its IV-PRICIT Program, and by the European Regional Development Fund (FEDER).
These striking phrases of [3]: "one of the great paradoxes of statistical mechanics is how a system can be energy conserving and deterministic on the (molecular) microscale, and yet dissipative and unpredictable/random on the macroscale. The paradox is resolved by recognizing that both the dissipation and the random fluctuations are associated with incomplete information about the state of the system." led us to use our A-model to put some physical sense in this noise-fluctuation field where some laws of Physics are infringed inadvertently. The A-model we found by using Thermodynamics to study noise in resistors [4] is implicit in the results of Johnson [6] and Nyquist [7]. The circuit it uses will be dealt with in Section 2. In Section 3 we will consider the example of [3] under our A-model to show the pitfall leading to the aforesaid paradox. Section 4 will show how the FDT should be understood and applied in today's research on electrical noise.

\section{A Physically Cogent Model for Electrical Noise in Resistors and Capacitors}

The origin of the FDT is the Quantum Mechanical treat- 
ment of a noisy system done by Callen and Welton [2] in 1951 where the need for a Complex Impedance (or Admittance) function to describe such a noisy system is so apparent (see Equations 2.12 to $2.15,3.6,4.6$ and 4.7 in [2]) that a paper like [1] transgressing this important result indicates that the Physical sense of Classical works like $[6,7,2]$ has been lost. The Admittance required by Thermodynamics in our A-model that agrees with the one required in [2] is the circuit shown in Figure 1. Although it reminds a circuit used today for noise in resistors, it has a capacitance $C$ (see Section VI of [4]) and it is more general because $R^{*}$ includes both ohmic and non-ohmic (e.g. differential) resistances that define the Conductance $G(\omega)=1 / R^{*}(\omega)$ or real part of the Admitance $Y(j \omega)=G(\omega)+j B(\omega)$ we will use to handle the noise voltage $v(t)$ as the time-varying signal linking noise current through the Conductance $G(\omega)$ with noise current through the Susceptance $B(\omega)$ at each frequency $f=\omega /(2 \pi)$.

Capacitance $C$ is required by Thermodynamics in order to have the energy store (Degree of Freedom) to have energy liable to fluctuate thermally in the device, linked with the fluctuating voltage observed as Johnson noise in resistors or $k T / C$ noise in capacitors. This unavoidable $C$ comes from the non null dielectric permittivity of any material (vacuum included) existing between two ohmic contacts or terminals put at some distance in our actual world [4,5]. The need to include in $R^{*}$ both ohmic and non-ohmic resistances also is dictated by Thermodynamics in order to keep the thermal fluctuation of $k T / 2$ Joules in the Degree of Freedom that $C$ represents. This meaning used in [8] to present the $k T / C$ noise as the noise coming from the Thermal fluctuation of $k T / 2$ Joules in the Degree of Freedom that $C$ represents, allows writing:

$$
\frac{1}{2} k T=\frac{1}{2} C\left\langle v^{2}(t)\right\rangle \Rightarrow\left\langle v^{2}(t)\right\rangle=\frac{k T}{C}
$$

thus showing the essential role of $C$ to generate noise in the circuit of Figure 1 and the secondary role of $R^{*}$ as a spectrum shaper to make (1) true [4,5]. This summarizes the advancing character of our A-model where the Johnson noise of resistors comes from their capacitance, a feature suggested in 1951 [2], but ignored in 2004 [1].

Considering any voltage noise in time $v(t)$ as synthesized from sinusoidal terms $v_{n}(t)$ (Fourier synthesis) each noise current $i_{P}(t)$ through the conductance $G=$ $1 / R$ linked with $v_{n}(t)$ will be a sinusoidal current in-phase with $v_{n}(t)$ given by: $i_{P}(t)=G \times v_{n}(t)$ (see Figure 2). The noise current $i_{Q}(t)$ linked with $v_{n}(t)$ will be a sinusoidal noise current in-quadrature with $v_{n}(t)$ given by: $i_{Q}(t)=j B \times v_{n}(t)$. For this positive $B$ due to $C$, a current $i_{Q}(t)$ will have $+90^{\circ}$ phase advance

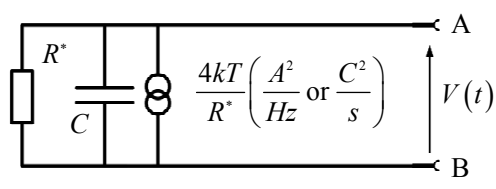

Figure 1. Complex Admittance used in the A-Model for the electrical noise of resistors and capacitors.

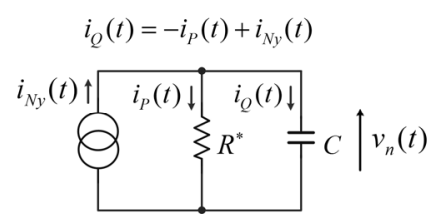

Figure 2. Electrical circuit that representing Johnson noise in resistors or $k T / C$ noise in capacitors, allows studying other noises obeying the Fluctuation-Dissipation Theorem.

respect to its sinusoidal $v_{n}(t)$. Therefore, the time integral of displacement currents like $i_{Q}(t)$ in $C$ will have the same phase of $v_{n}(t)$.

This coincidence of the aforesaid phases suggests, as our AM considers, that $i_{Q}(t)$ is the Cause that integrated in time by $C$, creates the Effect $v_{n}(t)$ that synthesizes the Johnson noise observed in resistors or the $k T / C$ noise observed in capacitors. This can be seen vividly in time domain, but this falls out of this paper and will be published elsewhere [9].

To deal with electrical noise due to Fluctuations (changes in time) of the content of electrical energy in two-terminal devices as resistors and capacitors, we will use the instantaneous power $p_{i}(t)=v_{n}(t) \times i(t)$, where $i(t)=i_{P}(t)+j i_{Q}(t)$ is the current through the device. Note that this same function (or better said, its average value $\left.\left\langle p_{i}(t)\right\rangle\right)$ was used in Equation 2.13 of [2]. To work in time domain, the Nyquist spectral density $4 k T / R$ $\mathrm{A}^{2} / \mathrm{Hz}$ derived from [7] $\left(4 k T / R^{*} \mathrm{~A}^{2} / \mathrm{Hz}\right.$ in our model to keep the $k T / C$ noise of $C[4,5])$ is replaced by its time counterpart: a random current $i_{N y}(t)$ with zero mean shown in Figure 2, whose Kirchoff's law on top we will write as:

$$
C \frac{\partial v_{n}(t)}{\partial t}=-G v_{n}(t)+i_{N y}(t)
$$

Equation (2) states that any band-limited $i_{N y}(t)$ will create a Displacement Current in $C$ and a Conduction Current in $R^{*}$. To create a pure Displacement current, a $\delta$-like current $i_{N y}(t)$ of infinite bandwidth $(\mathrm{BW} \rightarrow \infty)$ or null duration $\left(\delta t_{c} \rightarrow 0\right)$ is needed. The null time elapsed in this case $\left(\delta t_{c} \rightarrow 0\right)$ allows writing the time integral of (2) as:

$$
\Delta v_{\text {built }}=\frac{q}{C}
$$

where the weight $q$ of the $\delta$-like current $i_{N y}(t)$ (thus a 
charge) creates instantaneously a voltage step $\Delta v_{\text {built }}=q / C$ in $C$, or produces a Fluctuation of $q^{2} /(2 C)$ Joules in the energy of $C$ provided it was discharged. Thus, a pure Fluctuation of energy is not possible in resistors because their $i_{N y}(t)$ is band-limited as Nyquist showed by an upper limit for their $4 k T / R \mathrm{~A}^{2} / \mathrm{Hz}$ density due to Quantum Mechanical reasons [7].

Although very pure Fluctuations of energy result in this circuit for displacement currents with short $\delta t_{c}$, they always have a non null Dissipation due to the conduction current during the non null $\delta t_{c}$ elapsed. To say it bluntly: an electron leaving one plate with a kinetic energy of $q^{2} /(2 C)$ Joules exactly in a discharged $C$ is unable to create $\Delta v_{\text {built }}=q / C \mathrm{~V}$ in $C$ because part of its $q^{2} /(2 C) \mathrm{J}$ is dissipated during its non null transit time $\delta t_{c}$. Thus, no paradox exists if we find this system dissipative in the macroscale with a huge amount of such transits giving rise to noticeable dissipation of energy. Assuming, however, that these passages of single electrons between plates are energy-conserving events because the term $G v_{n}(t)$ has no time to dissipate a noticeable amount of energy during each short transit time $\delta t_{c}$, we would find a paradox due to this wrong assumption that we would have done for a parallel-plate capacitor in vacuum with plates at temperature $T$ before knowing that it is false due to the $R^{*}$ created by thermoionic emission [5]. This elemental charge noise of one electron in $C$ is the basis of [9] and the reason for the charge noise power units $\left(\mathrm{C}^{2} / \mathrm{s}\right)$ that appears in Figure $\mathbf{1}$ as equivalent (in time domain) to the well known $\mathrm{A}^{2} / \mathrm{Hz}$ units in frequency one.

\section{The Fluctuation-Dissipation Theorem and the A-Model for Electrical Noise}

Let us consider the Fluctuation-Dissipation Theorem explained by the motion of a large particle of mass $M$ through a sea of small particles having random motions [3]. These small particles exert, through collisions, a random force $F(t)$ on the large one whose velocity at time $t$ relative to the mean motion of the small ones is $v(t)$. The mean drag force on the large particle is proportional to $v(t)$ through the friction factor $\zeta$ and the fluctuating random component is proportional, with a coefficient of proportionality $\kappa$, to the so called statistical white noise $\Xi(t)$. Copying Equation (1) of [3], we have:

$$
M \frac{\partial v}{\partial t}=-\varsigma v+\kappa \Xi
$$

Following [3], "The aim of the fluctuation-dissipation theorem is to relate $\zeta$ to the statistics of $\kappa \Xi(t)$." Using a white noise process for $\Xi(t)$ the mean square fluctuating force $\left\langle F^{2}\right\rangle_{0}$ is obtained in Equation (10) of [3], which is:

$$
\left\langle F^{2}\right\rangle_{0}=\kappa^{2}\left\langle(\Xi(t))^{2}\right\rangle=\frac{2 k T \zeta}{\delta t_{c}}
$$

where $\delta t_{c}$ is the contact time during a collision. Below this Equation it is written: "This is the required statement of the fluctuation-dissipation theorem, relating as it does the mean square fluctuating force to the friction factor." [3].

Let us rewrite (2) in this form:

$$
C \frac{\partial v(t)}{\partial t}=-G v(t)+i_{N y}(t)=-G v(t)+\kappa \Xi
$$

where the noise current $i_{N y}(t)$ has been replaced by the product of a white noise $\Xi(t)$ and the constant $\kappa^{2}=k T \zeta$ given by Equation (9) of [3] to have a complete, formal analogy between (4) and (6). From this, the electrical counterpart of (5) is:

$$
\begin{aligned}
\left\langle i_{N y}^{2}(t)\right\rangle & =\kappa^{2}\left\langle(\Xi(t))^{2}\right\rangle=\frac{2 k T \zeta}{\delta t_{c}} \\
& =\frac{4 k T}{R^{*}} \times \frac{1}{2 \delta t_{c}} \approx \frac{4 k T}{R^{*}} \times f_{Q}
\end{aligned}
$$

where $\left\langle i_{N y}^{2}(t)\right\rangle$ has been approximated by the product of the flat density $S_{i}(f)=4 k T / R^{*} \quad \mathrm{~A}^{2} / \mathrm{Hz}$ by its bandwidth $f_{Q}(\mathrm{~Hz})$ derived from [7] as explained below.

Taking the equivalent bandwidth $f_{Q}$ for $S_{i}(f)$ from $k T \approx h f_{Q}$ (e.g. $f_{Q} \approx k T / h$ where $h$ is the Plank constant) (7) suggests that the interaction time behind the Nyquist noise of density $S_{i}(f)=4 k T / R^{*} \quad \mathrm{~A}^{2} / \mathrm{Hz}$ is: $\delta_{t c} \approx 0.08$ ps at room $T$. This $\left\langle i_{N y}^{2}(t)\right\rangle$ value that is not difficult to calculate (e.g. $\left\langle i_{N y}^{2}(t)\right\rangle \approx 10^{-10} \quad \mathrm{~A}^{2}$ or $10 \mu \mathrm{A}_{\text {rms }}$ at room $T$ for $R^{*}=1 \mathrm{k} \Omega$ ), is hard to be observed, however, being its time integral as $v(t)$ (Effect) all we will observe due to the unavoidable $C$ of any two-terminal device used to sense $i_{N y}^{2}(t)$ in our actual world. Leaving aside this reflection about the Measurement of this $i_{N y}^{2}(t)$ noise, let's go to the analogy between (2) and (4). Note the correspondences (voltage-velocity) and (current-force) we have because the (voltage $\times$ current) product is the instantaneous power $p_{i}(t)$ in the circuit due to the random noise generator $i_{N y}(t)$ (the time counterpart of $4 k T / R^{*} \mathrm{~A}^{2} / \mathrm{Hz}$ ) whereas the (velocity $\times$ force) product represents the instantaneous power $p_{i}(t)$ acting on the mass $M$ due to the sea of small particles hitting it along one spatial direction.

Therefore, results concerning Fluctuation and Dissipation of electrical energy that our A-model links with voltage noise of resistors (Johnson noise) and capacitors ( $k T / C$ noise), can be applied to the velocity noise of the large particle of mass $M$. The first one we will use is the 
impossibility of energy-conserving collisions whose collision time is not null $\left(\delta t_{c}>0\right)$ to keep finite the interaction power. Due to this, the system of [3] can't be taken as "energy conserving and deterministic on the microscale" as it was done in the premise of the paradox at hand. The dissipative action of the $\zeta v$ term of (4) never vanishes for $\delta t_{c}>0$ (except for $\zeta=0$, which is not the case). Thus, the paradox comes from its false premise. We found a similar paradox or hypothetical device in [5] trying to build a pure capacitance $C$ in Thermal Equilibrium (TE) at some temperature $T$ because thermoionic emission is enough to create a differential resistance $R^{*}$ shunting $C$ to form an energy relaxing cell bearing the Admittance that Quantum Mechanics requires for a noisy system [2].

Therefore, the proposal of a noisy resistor offering a pure Resistance $R$ in TE at some temperature $T$ [1] does not make physical sense because it is a hypothetical device that infringes Thermodynamics and Quantum Physics. Without Susceptance, this device is unable to store electrical energy liable to fluctuate or it lacks the eingenstates of electrical energy required in [2] to allow a Quantum treatment of noise. Even more: the hypothetical device of an $R$ without $C$ infringes Special Relativity too because the null dielectric permittivity of the material required to have $C=0$ between two neighbour terminals would allow the speed of the light to be boundless in it [5].

Once solved the paradox recognizing that it comes from a wrong premise that would require an infinite interaction power, let us say that since Fluctuation is linked with a current in quadrature respect to $v_{n}(t)$ and Dissipation is linked with a current in phase, the phase between observed Effect and its Cause is what defines if energy mostly Fluctuates (as electrical energy in $C$ or kinetic energy of $M$ ) or if it is being Dissipated (by a current $i_{P}(t)=v_{n}(t) / R^{*}$ in the device of Figure 2 or by the drag force $\zeta v(t)$ in [3]). The study of the example handled in [3] could benefit from these ideas by using the circuit of Figure 2 as the mechanical impedance $Z(j 2 \pi f)$ or transfer function velocity/force at each frequency of the large particle in the viscous sea of small ones. Thus, let us consider the large particle moving back and forth due to a sinusoidal force $F(j 2 \pi f)$ as one of the Fourier components of the random force $\kappa \Xi(t)$ in (4). At low frequencies, the term of (4) proportional to the time derivative of the velocity $v(j 2 \pi f)$ is small, the Cause $F(j 2 \pi f)$ and the Effect $v(j 2 \pi f)$ tend to be sinusoids in-phase and thus, the energy brought by the force $F$ as time passes is mostly dissipated by the friction represented by the drag force $\zeta v(t)$. The inertial storage of energy by low- $f$ components of $F$ acting on $M$ is small, thus being Dissipation-like forces because Cause
$(F)$ and observed Effect $(v)$ tend to be in-phase.

At high- $f$, however, where $f \gg f_{C}=\zeta /(2 \pi M)$, the transfer of energy to and from the mass $M$ accelerated sinusoidally dominates over the energy spent against the drag force $\zeta v(t)$. This tends to put the sinusoidal force $F(j 2 \pi f)$ with a phase advance of $+90^{\circ}$ respect to the sinusoidal velocity $v(j 2 \pi f)$. Cause (Force) and its Effect (velocity) tend to be in quadrature and thus, high-frequency components of forces acting on $M$ will be Fluctuation-like forces. Collisions where a small particle moves fast respect to the large mass $M$ it hits during $\delta t_{c}>$ 0 will produce a brief force packet whose Fourier components mostly will be of this type. This allows to consider these collisions as Fluctuation-like or very elastic ones, but not as energy-conserving collisions because dissipation never vanishes due to their collision time $\delta t_{c}>0$ needed to have a finite interaction power.

Besides the above, the velocity distribution of the small particles in [3] leads to find some of them with very low velocities relative to $v(t)$. A small particle and the large one approaching at a few nanometres per year not only suggest a larger collision time or an enhancement of the role of the time integral of the real part of $p_{i}(t)$ respect to the role of its imaginary part, but also they suggest another reason avoiding elastic collisions. At this low relative velocity, the large and the small particles can join in the collision by internal forces as gravitational ones, thus having an inelastic collision of very long $\delta t_{c}$ where the conservation of momentum leads to lose energy as it is known from textbooks. This effect due to internal forces overseen at first sight would change the mass $M$ and the simplicity of the problem, of course, but illustrates the weakness of the premise about energy-conserving collisions between masses. It also illustrates vividly the drag action of the large mass $M$ on the small particles at low relative velocity in a way that the nearly zero-phase relation between sinusoidal Cause $(F)$ and Effect $(v)$ does not suggest so clearly.

\section{On the Application of the Fluctuation-Dissipation Theorem in Noise Physics}

The proper application of the FDT in Physics requires knowing its physical meaning. Equations (5) and (7) show that the FDT relates the mean square fluctuating force to the friction factor or the mean square fluctuating current to the Conductance $G=1 / R^{*}$ with no reference to the Degree of Freedom allowing Fluctuations of energy (mechanical or electrical). The mass (inertia) or the capacitance are irrelevant provided they exist in the system (e.g. $M>0, C>0$ ) because once the Degree of Freedom exists, its $k T / 2$ thermal fluctuation will define the ob- 
served noise in TE. This becomes apparent when one "reads" the circuit of Figure 1 with Thermodynamics in mind: the suspicious dependence of the noise density $S_{i}(f)=4 k T / R^{*} \mathrm{~A}^{2} / \mathrm{Hz}$ on the resistance $R^{*}$ it is going to drive, is the required one to have a mean square voltage noise $\left\langle v^{2}(t)\right\rangle=k T / C \mathrm{~V}^{2}$ independent of $R^{*}$ for the common case of circuits where $f_{c}=1 /\left(2 \pi R^{*} C\right) \ll f_{Q}$. Since $f_{Q}>6 \mathrm{THz}$ at room $T$, any stray capacitance added to the $C_{d}=\tau_{d} / R$ of a resistor [4] is enough to make true this $f_{c} \ll f_{Q}$ condition where the noise density $S_{v}(f)=4 k T R^{*} \times\left(R^{*}\right)^{2} \quad\left(S_{v}(f)=4 k T R^{*} \mathrm{~V}^{2} / \mathrm{Hz}\right)$ is proportional to $R^{*}$ whereas the circuit bandwidth is inversely proportional to $R^{*}$. This means that $R^{*}$ has nothing to do with the integral of $S_{v}(f)$ from $f \rightarrow 0$ to $f \rightarrow \infty$, which is: $\left\langle v^{2}(t)\right\rangle=k T / C \mathrm{~V}^{2}$, thus indicating that the root mean square (rms) voltage noise does not depend on $R^{*}$, but on $C$. Hence, Fluctuations of energy in $C$ are the mechanism that generates the Nyquist noise $S_{i}(f)=4 k T / R^{*} \mathrm{~A}^{2} / \mathrm{Hz}$ of a resistor, thus giving its advancing character to this A-model for electrical noise contending that the circuit element that generates the Johnson noise in resistors is their capacitance. The complete view in time domain of these Fluctuations of electrical energy followed by Dissipations of the energy unbalance set by each Fluctuation is given in [9].

Therefore, the FDT relates the mean square fluctuating force (voltage) to the friction factor (conductance) in a noise system provided fluctuations of energy can take place in it. The non-null mass $M$ (or inertia) of the large particle sets this Degree of Freedom in [3] that in our A-model is due to $C$. This is why we found hard to apply the FDT to the pure resistance of [1], whose $C=0$ (e.g. a large particle of $M=0$ in [3]), poses some troubles because the collision of a small particle with a "big one" of null mass wouldn't be a collision (e.g. no transfer of kinetic energy nor momentum would take place). This non-sense situation for $M=0$ shows the non-sense proposal of [1] with $C=0$, where only instantaneous fluctuations of energy (e.g. $\delta t_{c}=0$ ) could store some energy in such "device", fluctuations that do not exist [7]. Note that the assumption of this sort of "energy-conserving" fluctuations in [3] $\left(\delta t_{c}=0\right)$ led to the aforesaid paradox.

To conclude we will say that words like: totally elastic, energy conserving, totally dissipative, pure resistance, pure capacitance and so on must be used with care because they can exclude other phenomena that are essential to understand the problem at hand. This is so for magnitudes like electrical power that depending on the existence in time and on the change with time of the electrical voltage has two orthogonal terms, none of which describes completely the noisy system. Appendix I shows active and reactive power as two orthogonal terms of instantaneous power linked with Dissipation and
Fluctuation of electrical energy in resistors and capacitors. It is worth reading where the electrical energy fluctuating thermally was stored in the compound device Nyquist used to apply Thermodynamics [7]. It was stored in the Susceptances of a lossless Transmission Line, whence it may be seen that two opposed susceptances (capacitive and inductive) cancelling mutually at frequency $f_{0}$, don't cancel their ability to store electrical energy at this $f_{0}$. Considering the need for this ability to store energy in noisy systems as it appeared in the Classical works of Nyquist and Callen \& Welton, we will recover the Physical sense in this Fluctuation-Dissipation field where electrical noise is perhaps its best known exponent. And to end this paper showing the agreement between Quantum Physics and Classical Thermodynamics in the noise field we will say that this kind of agreement also appears in other fields of Physics [10].

\section{Acknowledgements}

We wish to thank Prof. E. Iborra, head of the GMME, for encouraging chats about the meaning of "well known" in research. We also thank Prof. H. Solar for his cordial welcome to give a talk on these ideas at CEIT, in the Universidad de Navarra.

\section{References}

[1] G. Gomila, C. Pennetta, L Reggiani, M. Sampietro, G. Ferrari and G. Bertuccio, "Shot Noise in Linear Macroscopic Resistors," Physical Review Letters, Vol. 92, No. 22, 2004, pp. 226601-226604. doi:10.1103/PhysRevLett.92.226601

[2] H. B. Callen and T. A. Welton, "Irreversibility and Generalized Noise," Physical Review, Vol. 83, No. 1, 1951, pp. 34-40. doi:10.1103/PhysRev.83.34

[3] P. Grassia, "Dissipation, Fluctuations, and Conservation Laws," American Journal of Physics, Vol. 69, No. 2, 2001, pp. 113-119. doi:10.1119/1.1289211

[4] J. I. Izpura, "1/f Electrical Noise in Planar Resistors: The Joint Effect of a Backgating Noise and an Instrumental Disturbance," IEEE Transactions on Instrumentation and Measurement, Vol. 57, No. 3, 2008, pp. 509-517. doi:10.1109/TIM.2007.911642

[5] J. I. Izpura, "On the Electrical Origin of Flicker Noise in Vacuum Devices," IEEE Transactions on Instrumentation and Measurement, Vol. 58, No. 10, 2009, pp. 3592 3601. doi:10.1109/TIM.2009.2018692

[6] J. B. Johnson, "Thermal Agitation of Electricity in Conductors," Physical Review, Vol. 32, No. 1, 1928, pp. 97-109. doi:10.1103/PhysRev.32.97

[7] H. Nyquist, "Thermal Agitation of Electric Charge in Conductors," Physical Review, Vol. 32, No. 1, 1928, pp. 110-113. doi:10.1103/PhysRev.32.110 
[8] D. A. Bell, "Noise and the Solid State," Pentech Press, London, 1985.

[9] J. I. Izpura and J. Malo, "A Fluctuation-Dissipation Model for Electrical Noise," Circuits and Systems, Vol. 2, No. 3, 2011, pp. 112-120.

\section{Appendix I}

In the circuit of Figure 2, for a sinusoidal $v_{n}(t)$ the current $i_{P}(t)$ is sinusoidal whereas the current $i_{Q}(t)$ is a cosine current advanced $+90^{\circ}$ respect to $v_{n}(t)$. Therefore, the instantaneous power $p_{i}(t)$ delivered by the generator $i_{N y}(t)$ in Figure 2 has an Active power always positive $p_{i P}(t)=v_{n}(t) \times i_{P}(t)$ and a Reactive power $p_{i Q}(t)=v_{n}(t) \times i_{Q}(t)$ entering and leaving periodically the Susceptance of the device (e.g. oscillating at $2 f$ that is the way a Susceptance stores electrical energy at a given frequency $f$ ). If, and only if, this storage exists in the device, the stored energy can Fluctuate in it. Let us show how Active and Reactive power are, respectively, Dissipation of electrical energy in $R$ without storage and Fluctuation of electrical energy in $C$ without dissipation. Let's consider $v_{n}(t)=A \times \sin (\omega t)$ in Figure 2, where the angular frequency $\omega$ is $2 \pi$ times the natural frequency $f: \omega=2 \pi f$. From this $v_{n}(t)$ existing in $C$ and from the current it produces in it: $i_{Q}(t)=C \times\left(\partial v_{n}(t) / \partial t\right)$, the instantaneous power $p_{i Q}(t)$ entering $C$ is:

$$
\begin{aligned}
p_{i Q}(t) & =A \sin (\omega t) \times(A \omega C) \cos (\omega t) \\
& =\left(A^{2} \omega C\right) \times \sin (\omega t) \times \cos (\omega t)
\end{aligned}
$$

On the other hand, the fluctuating electrical energy being stored in $C$ by $v_{n}(t)$ will be:
[10] T. Miyashita, "Quantum Physics Can Be Understood in Terms of Classical Thermodynamics," Journal of Modern Physics, Vol. 2, No. 1, 2011, pp. 26-29. doi:10.4236/jmp.2011.21005

$$
\begin{aligned}
& U_{E}(t)=\frac{C}{2} \times(A \sin (\omega t))^{2} \Rightarrow \\
& \frac{\partial U_{E}}{\partial t}=\left(A^{2} \omega C\right) \times \sin (\omega t) \times \cos (\omega t)
\end{aligned}
$$

From (8) and (9) we conclude that the instantaneous power entering $C$ is used to vary (fluctuate) the electrical energy stored in $C$. No part of $p_{i Q}(t)$ is dissipated, thus linking Fluctuations of electrical energy in Resistors or Capacitors with currents in quadrature with $v_{n}(t)$ whereas Dissipations of electrical energy in these devices will come from currents in-phase with $v_{n}(t)$. From the current $i_{P}(t)=v_{n}(t) / R^{*}$ that $v_{n}(t)$ produces in the resistance $R^{*}$, the instantaneous power $p_{i P}(t)$ entering $R^{*}$ is:

$$
\begin{aligned}
p_{i P}(t) & =A \sin (\omega t) \times \frac{A}{R^{*}} \sin (\omega t) \\
& =\frac{A^{2}}{R}(1-\sin (2 \omega t))
\end{aligned}
$$

Contrarily to (9) whose mean value is null because energy entering $C$ is released subsequently, energy always enters $R^{*}$ because (10) is a positive power of mean value $P_{\text {avg }}=A^{2} /(2 R)$. All the instantaneous power entering $R$ is thus dissipated and no part of it is stored. 